\title{
Leg loop Harness. Design and Method 6
}

\section{Alfonso Godino}

This method has been used on Bearded Vultures Gypaetus barbatus, Cinerous Vulture Aegypius monachus, Eurasian Griffon Vulture Gyps fulvus, Rüppell`s Vulture Gyps ruepelli, Short-toed eagle Circus gallicus, Red Kite Milvus milvus, Montagus' Harrier Circus pygargus, Bonelli's Eagle Aquila fasciata and Golden Eagle Aquila chrysaetos in Spain, Portugal, Italy and Morocco.

With the Bearded Vulture, Cinereous Vulture and Red Kite, individuals tagged included full-grown birds and nestlings, where a potential size increase could still occur after the tagging in this last group. For other species, only full-grown individuals were tagged with this method.

Devices used include: Aquila 33g, Anitra 50g, BTO 25g, Microwave 45g, Ornitela 50, 25 and $10 \mathrm{~g}$, Vectronic $90 \mathrm{~g}$. http://dx.doi.org/10.4314/vulnew.v78ai1.10

\section{Materials and Equipment needed}

- Tubular Teflon ribbon: $4.1 \mathrm{~mm}(0.16 ")$ and $6.4 \mathrm{~mm}$ wide ( 0.25 ") for small-medium species (Milvus, Circus) and $8.4 \mathrm{~mm}$ wide (0.33") for bigger (vultures, eagles, etc.)

- For expandable or elastic harness, a clothing elastic to insert in the Teflon (0.08"/2mm narrower than Teflon used).

- Cyanocrylate (superglue)

- Cyanocrylate activator spray

- $10 \times 6 \mathrm{~cm}$ rough/porous pad (scouring or neoprene pad are useful)

- Neoprene pad to glue to the lower part of the device (if not supplied by the transmitter's manufacturer)

- Plastic needle

- Scissors

- Clamp scissors - straight and curved

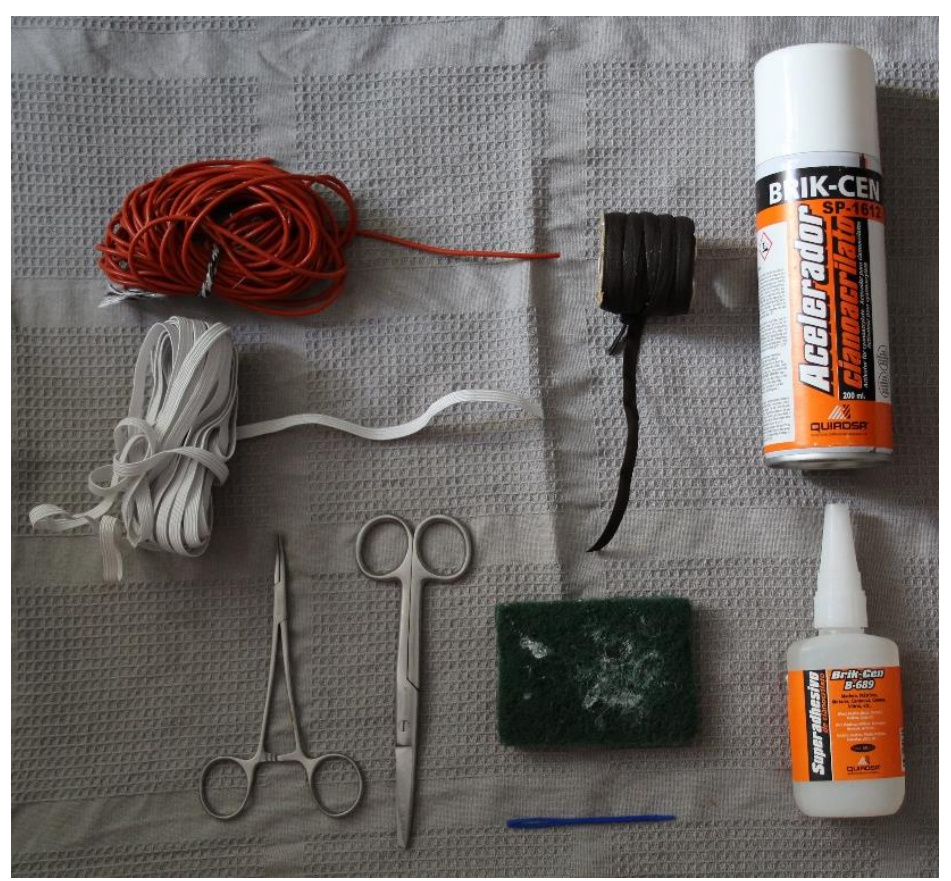

Figure 6.1: Equipment 


\section{Measurements and preparations: Key stages/steps}

Here I present the harness used to fit two types of devices, one model with a tube/hole in front and back of the device (A) and the second one mounted on a device with one lug in front and two lugs on the back-lateral side (B) (Fig 6.2)

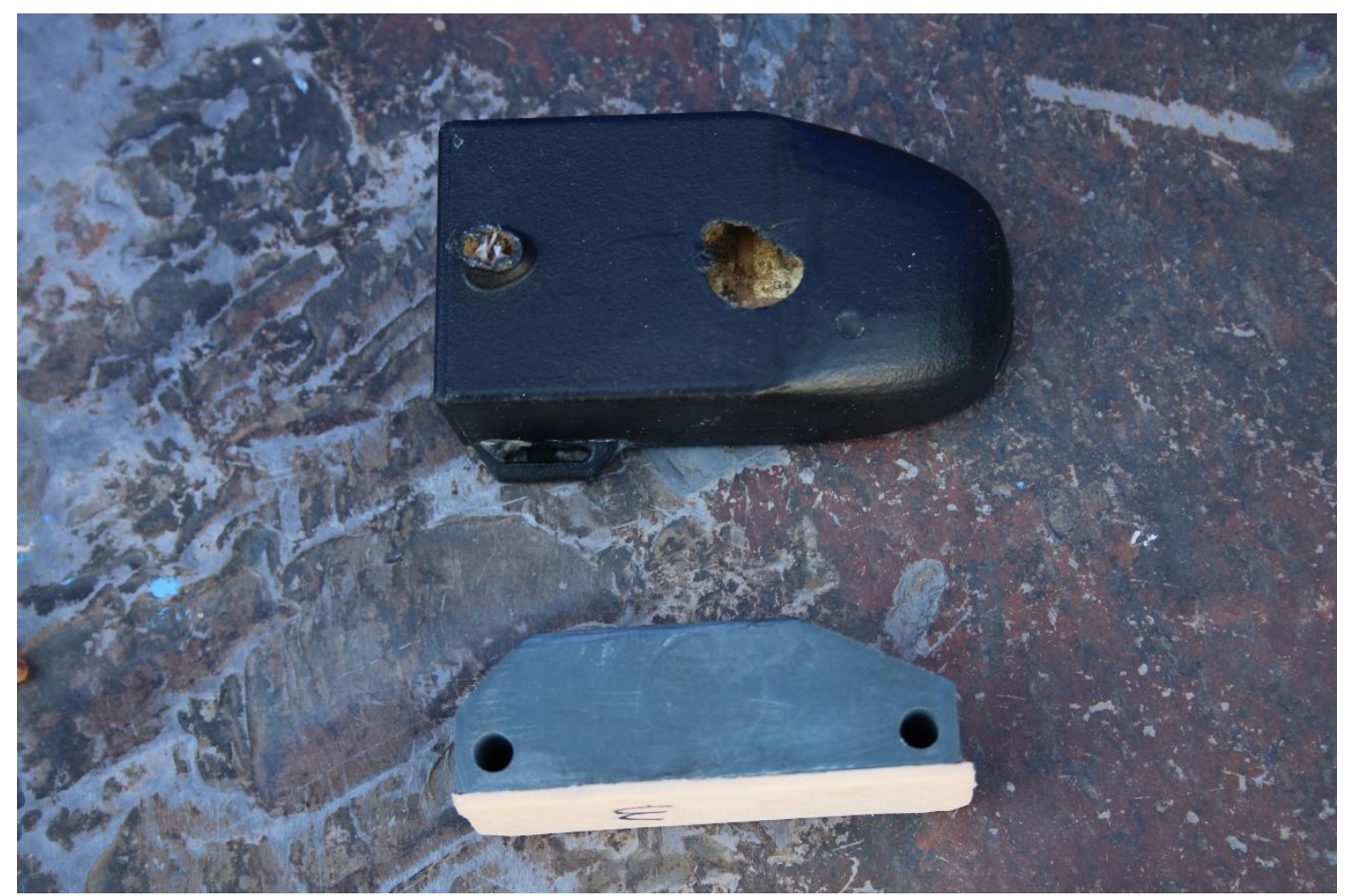

Figure 6.2:

It should be easy to adapt these two methods for different transmitters with other attachment lugs.

Both methods allow the use of expandable and non-expandable harness.

The attachment of the harness to the device is made before fitting it to the bird, with the goal of reducing the time spending handling the bird.

If you have no experience with the species, it is highly recommended to test any harness in captivity (rehabilitation and breeding in captive centres, zoos, falconry shows/exhibitions, private collections, etc), before using on a wild bird, or to be trained by more experienced practitioners on the given method and species.

\section{A. Device with hole/tubes in front and back}

The Teflon tape will be in one piece (length will depend with the species) and, to facilitate the insertion through the holes/tubes, tips of the Teflon are cut sharply. 


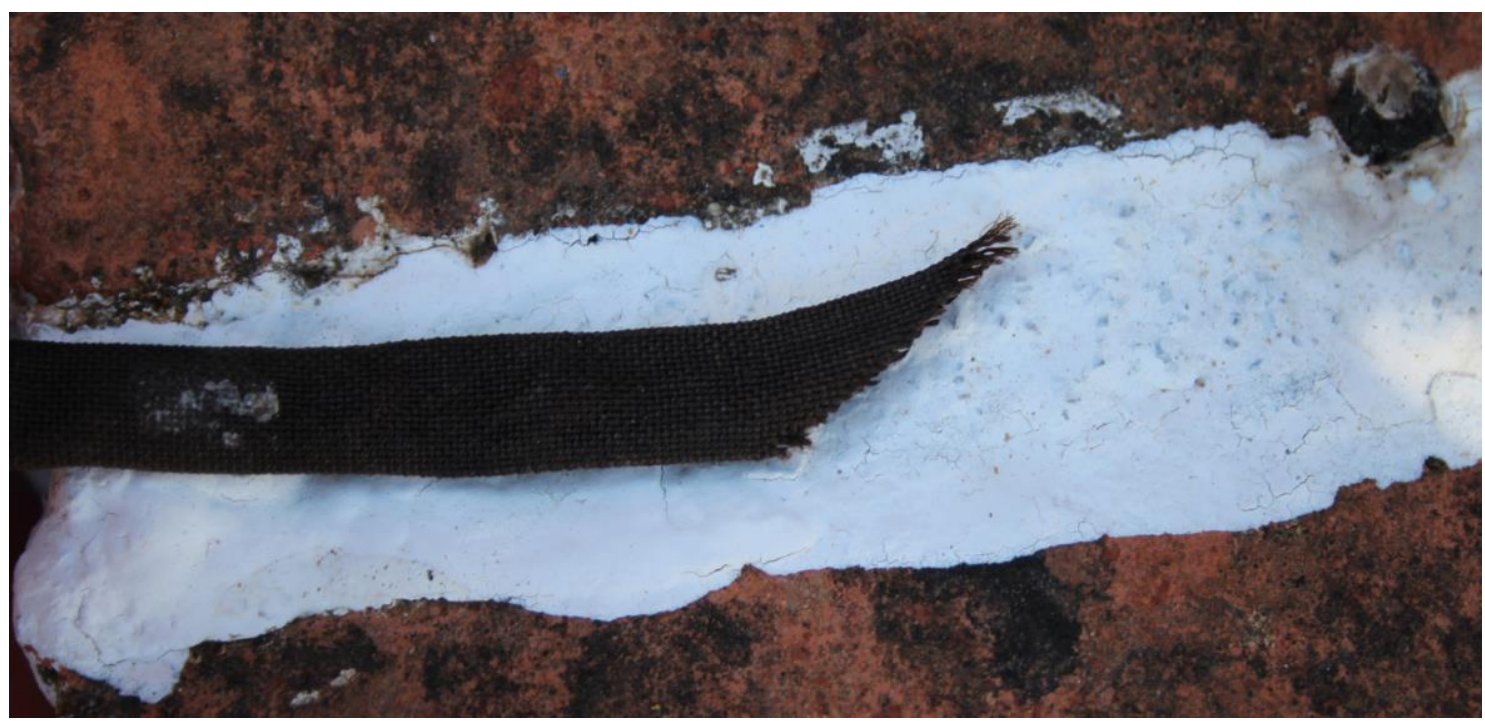

Figure 6.3

Additionally, and to avoid the fraying of the tip, a little superglue can be added to the tip. With the glue, the tip will be stiff and this will make it easier to pass the Teflon through the holes/tubes. Pass the Teflon through the front hole/tube and be sure the length is equal on both sides.

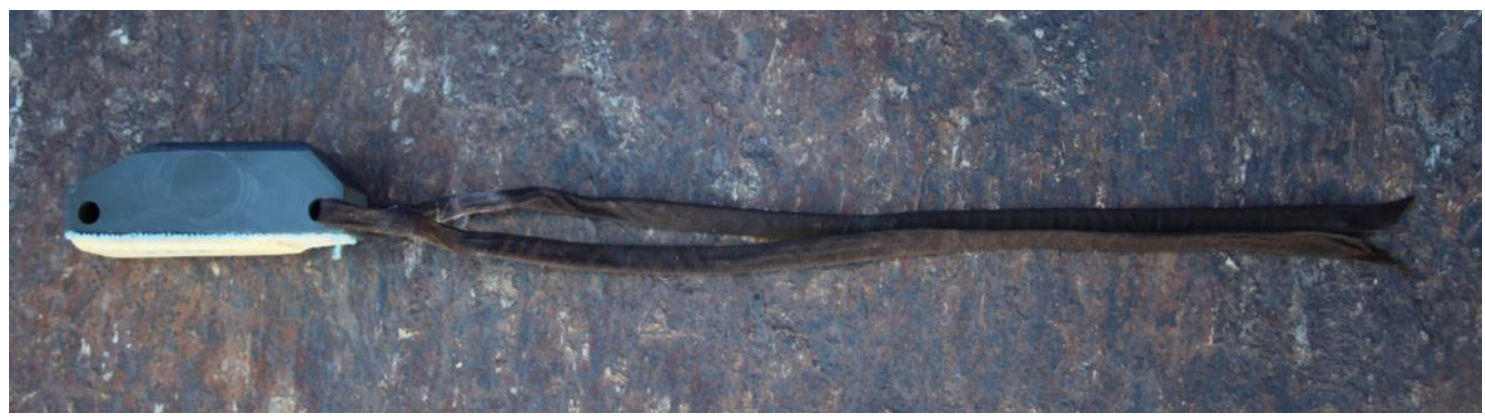

Figure 6.4

Then make a knot as close to the device as you can.

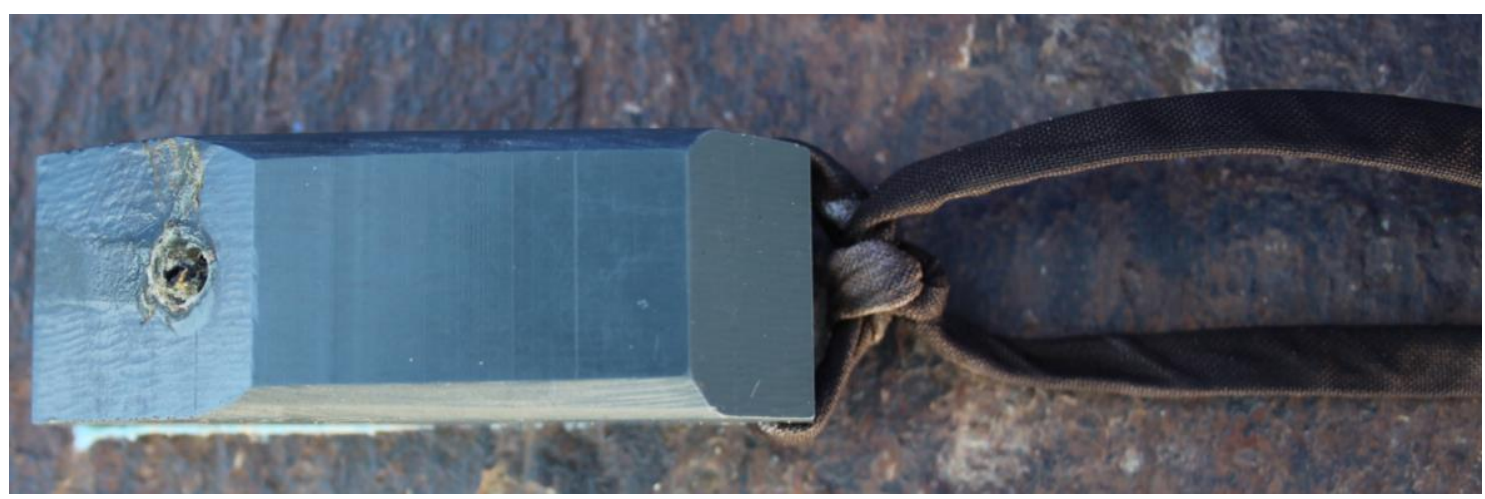

Figure 6.5 
Before tying the knot, add glue inside the knot, and again add glue around the knot once tied. To make the glue dry faster use cyanoacrylate activator spray.

Pass both Teflon tips through the back hole/lug. When both tips are passed though the holes/tubes, knot them on the back side of the device.

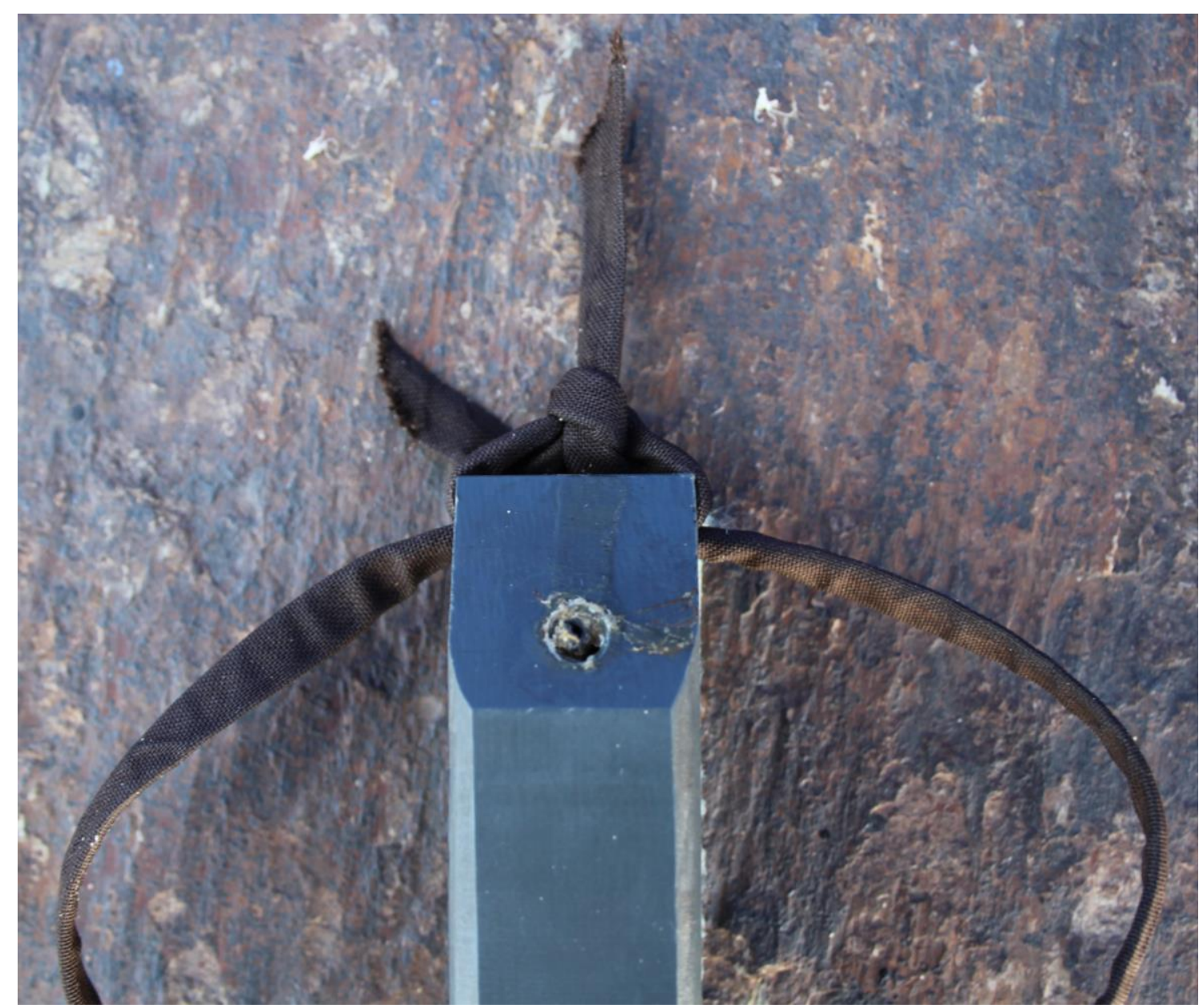

Figure 6.6

This knot should not be very tight, since we will need to tie off later when attached it to the bird. If you prefer the knot in front of the device and not in the back, the process is the opposite, inserting the Teflon through the back hole/tube and keep the knot in the front.

\section{B. Device with one lug in front and two lugs on the back-lateral side}

As previously, Teflon tape will be in one piece (length will depend with the species) and to facilitate the insertion through the lugs, tips of the Teflon must be cut sharply (Fig 6.3) and glue can be added to the tip.

Pass the Teflon through the front lug and be sure the length is equal on both sides (Fig 6.4). Then make a knot as close to the device as you can. 


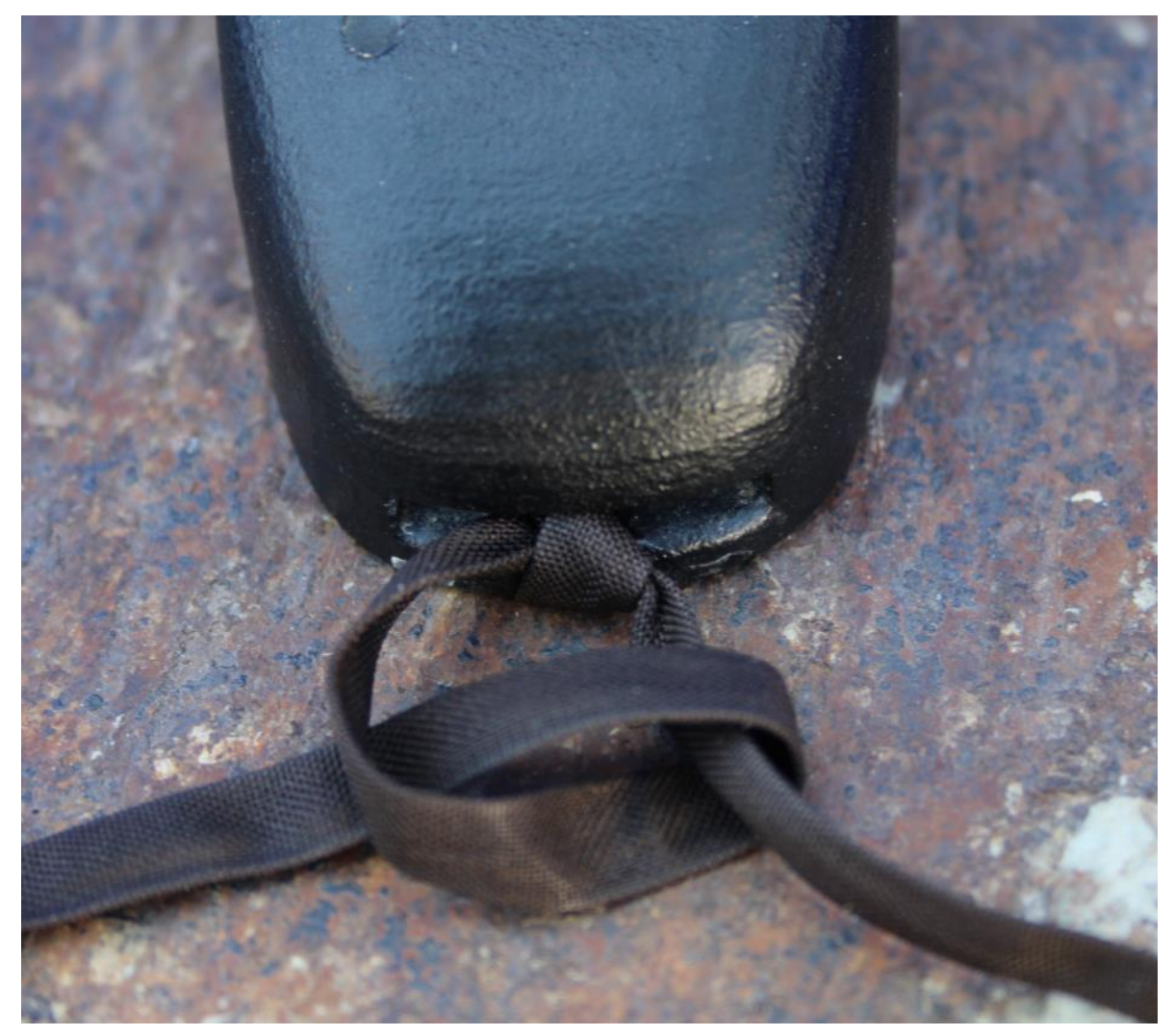

Figure 6.7

Before tying the knot, add glue inside the knot, and again add glue around the knot once tied. To make the glue dry faster use cyanoacrylate activator spray.

Pass both Teflon tips through each one of the lugs and knot them on the back side of the device.

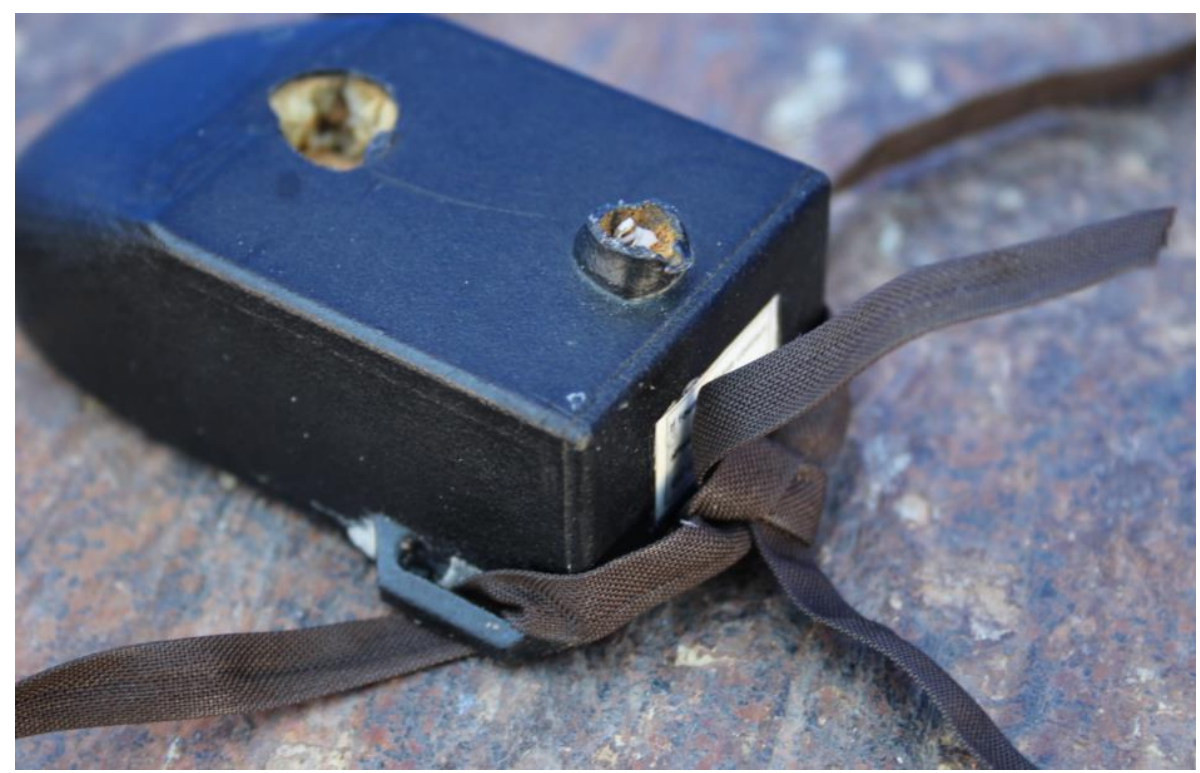

Figure 6.8 
This knot should not be very tight, since we will need to tie off later when attached it to the bird.

With the harness attached to the device, on both models, you can glue the neoprene pad on the base of the transmitter. Care must be taken to not spread glue too close to the corners where hole/tubes or lugs are.because it could make difficult when we adjust the harness on the bird (neoprene pad could be glued to the Teflon ribbon).

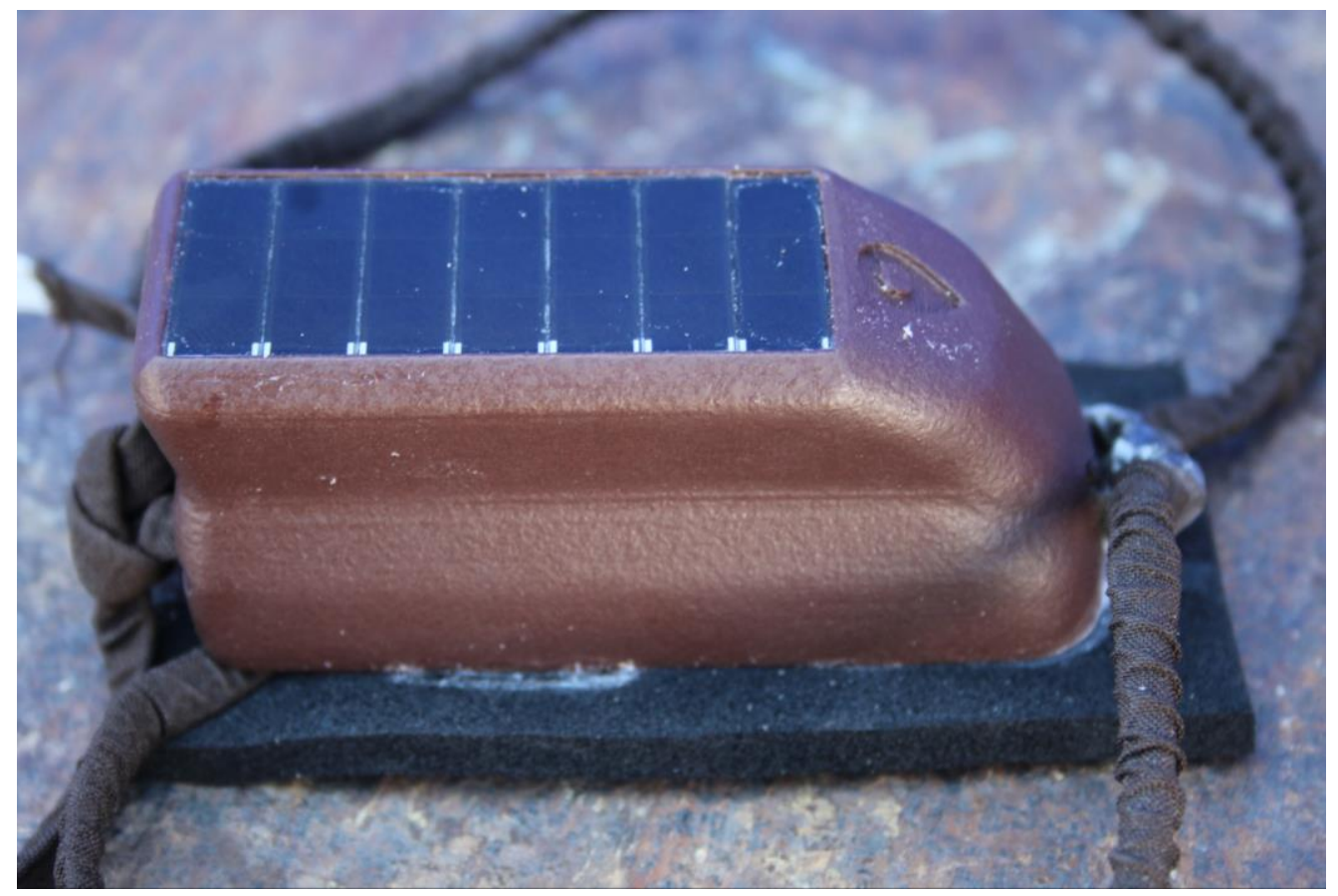

Figure 6.9

\section{Expandable harness}

To make expandable on both methods previously described, a clothing elastic or silicone cord can be inserted into the Teflon ribbon. Recently we have been using clothing elastic as an alternative to the silicone cord for several reasons: it is cheaper, it is easier to get and it is much easier to insert into the Teflon ribbon. To insert the clothing elastic inside the Teflon, a plastic needle is used to avoid damaging the Teflon.

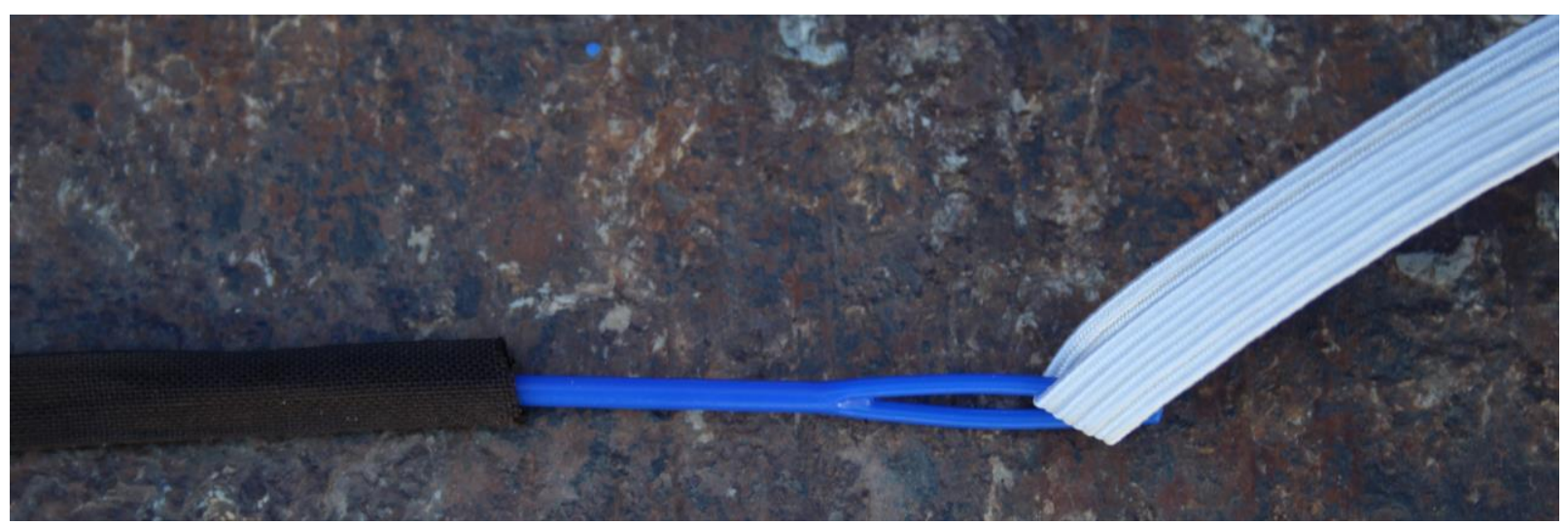

Figure 6.10 
It is advisable to use longer elastic than Teflon ribbon. Normally $6-10 \mathrm{~cm}$ will be enough. With this measurement, the harness will have $3-5 \mathrm{~cm}$ of extra elastic in each part.

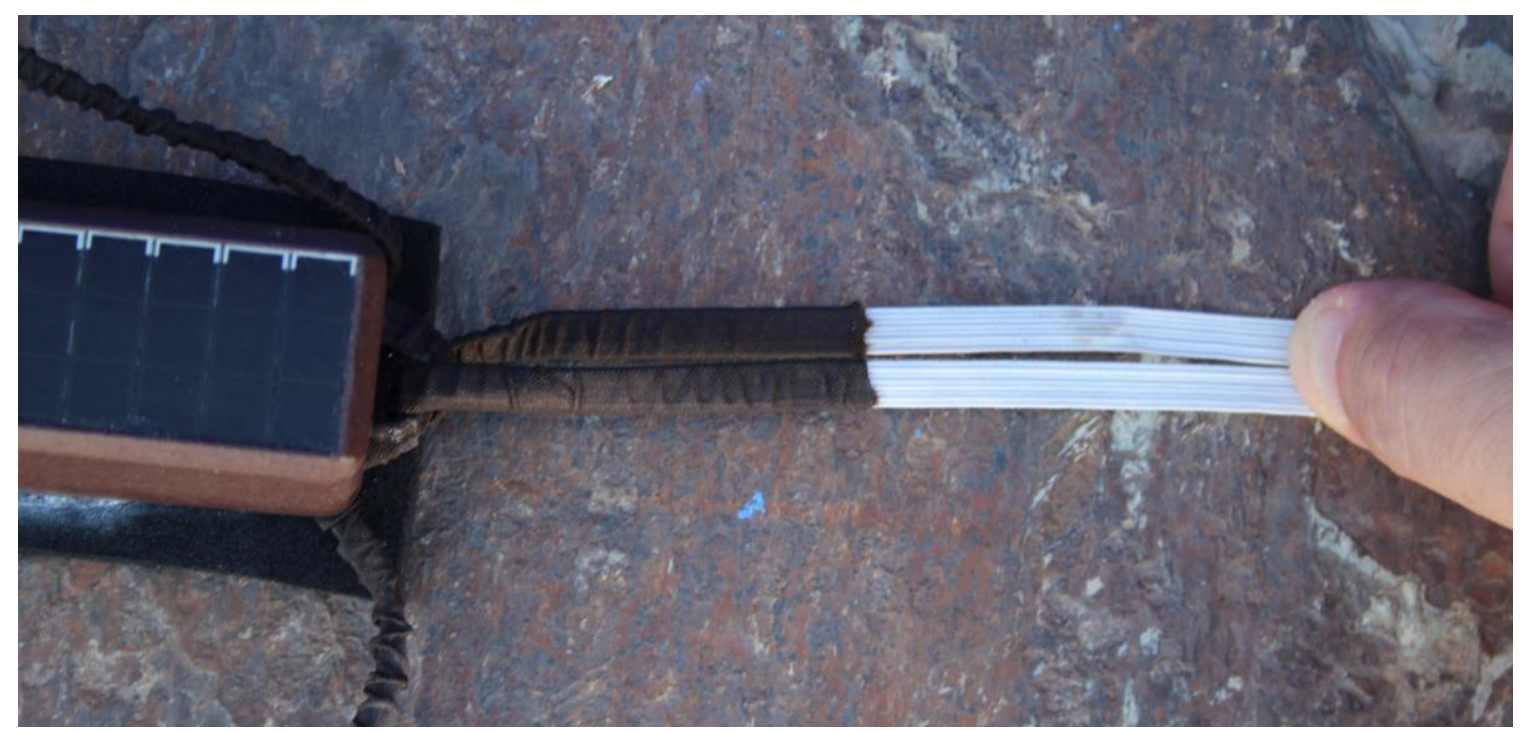

Figure 6.11

With the elastic inside the Teflon, follow the steps shown previously in A and B.

\section{Restraint and hooding}

Normally two persons are needed, one person to hold the bird and the second to fit the harness. Only in bigger and more active species, such as Gyps, a third person is recommended to help hold the bird. To keep the bird calm, the use of a hood designed for raptors is recommended. There are hood designs for all vulture species and many other raptors, even for those species where due to the morphology of the head and neck, as Gyps species, it may be difficult for the bird to wear a hood.

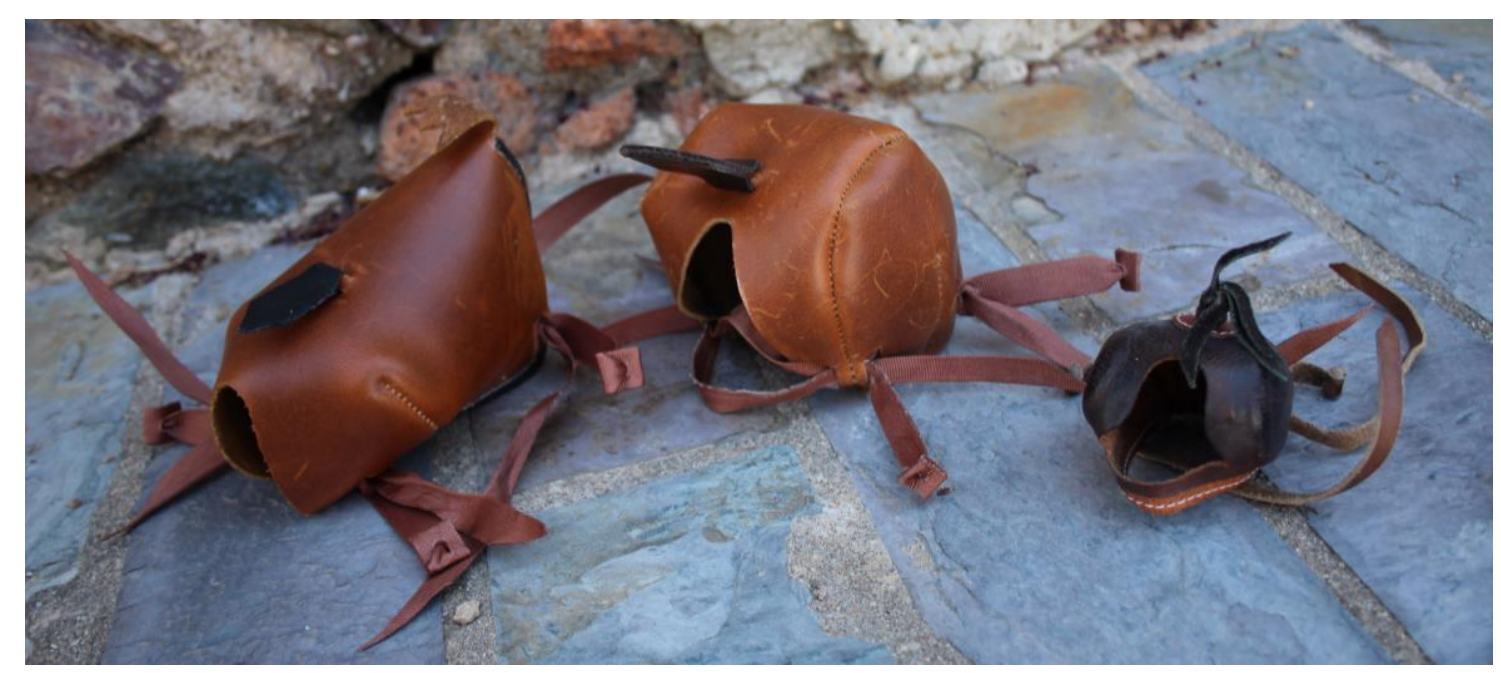

Figure 6.12 
The advantage of the hood is that it is easy to put it The collaboration of a wildlife veterinarian during on and remove it, it does not touch the eyes as socks would do. If the same hood is used with several birds (several individuals trapped at the same time and one or few hoods), it must be disinfected when used on a new bird, especially the inner part (e.g. disinfectants with quaternary ammonium and biguanidine are very effective, non-toxic, non-irritating, non-corrosive and biodegradable). trapping, handling and tagging is also an advisable precaution against potential problems, especially when you plan to trap/tag several birds at the same time.

Birds must be kept with the breast on the ground, if possible on a soft or padded carpet and in shade. For small and medium size species, e.g. Circus, Falco, it is easier to keep them in the hand during the handling and tagging (Fig 6.13).

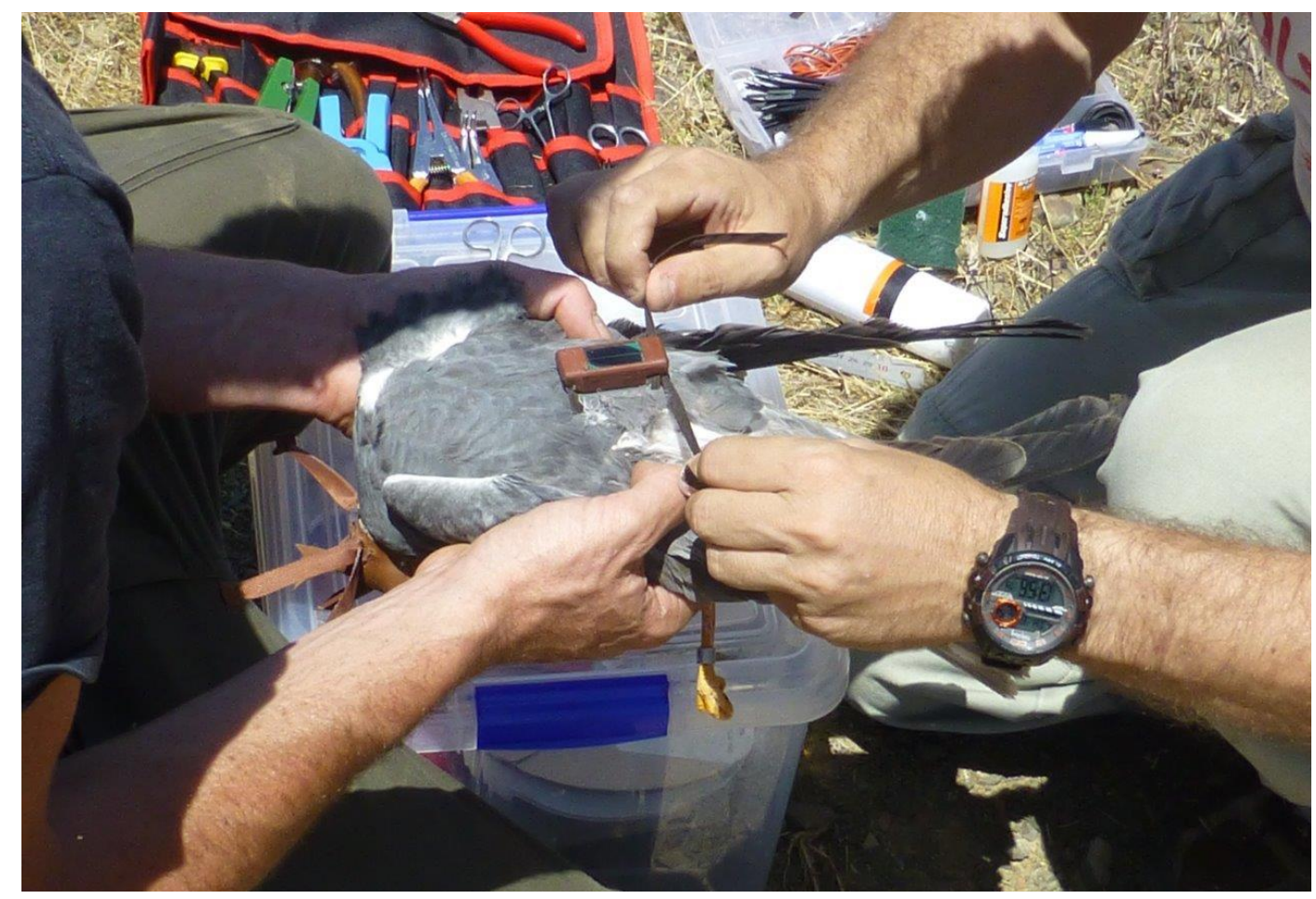

Figure 6.13

\section{Fitting to the bird}

\section{Non-elastic harness}

1) With the breast on the ground, the bird will stay in the same position and you can place the device on the back. Tie off the knot on the back side of the transmitter and pass each ribbon in front of the knee and under the leg to recover the tip by the back of the leg. Insert the ribbon through the hole/tub or lug. After passing the ribbon, hold both tips together using clamp scissors. 


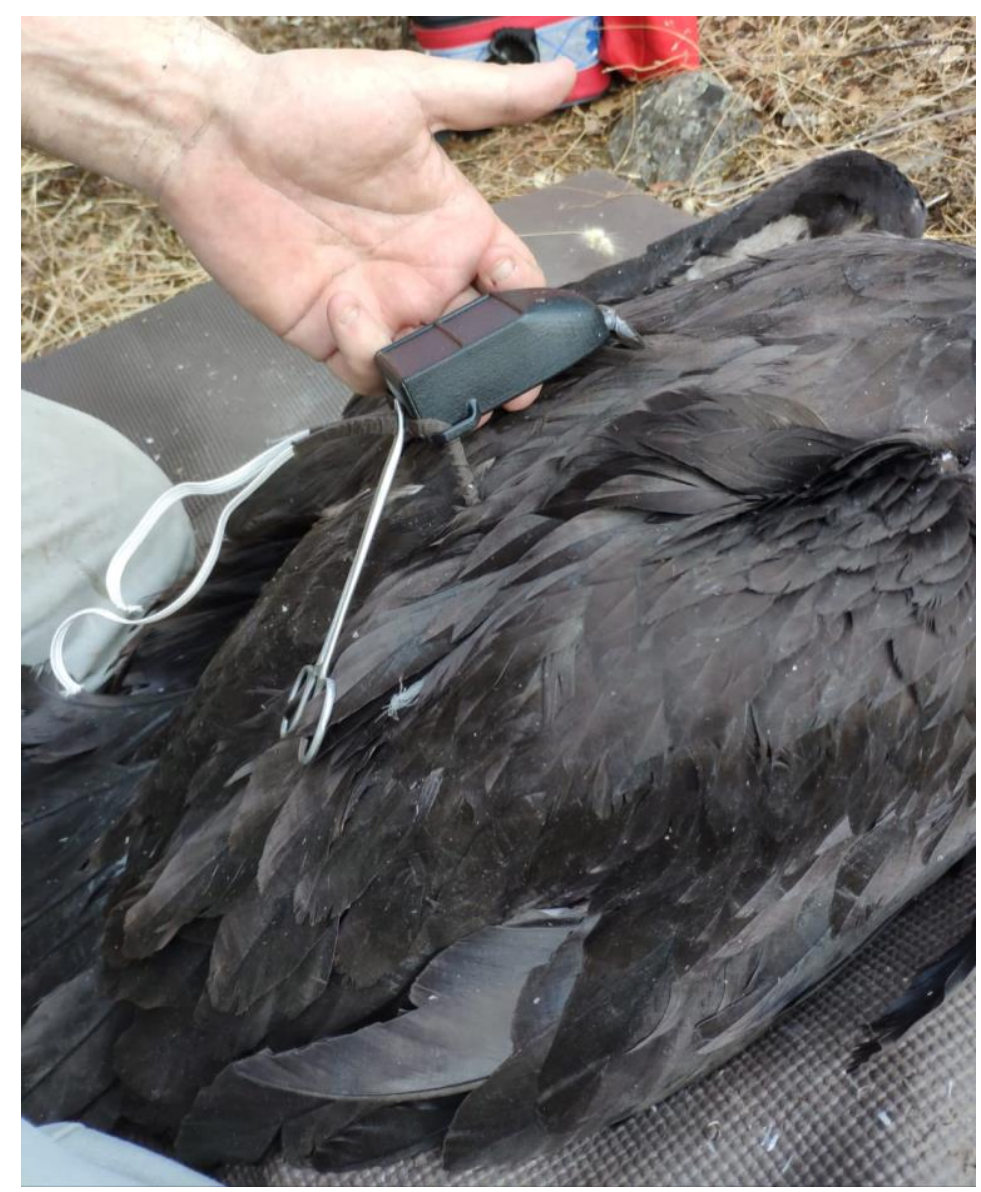

Figure 6.14

In this situation you can adjust the harness until you get the correct fit (paying attention to keep the same ribbon length). Check the correct adjustment by passing the fingers in between the ribbon and the body all along the ribbon. Check the presence of feathers: tertials, axillaries and thigh, in between the ribbon and the body. No feathers must be trapped in this position.If you prefer the knot in the front side of the transmitter, in the devices with a hole/tube, the process is the opposite as described. You must keep the knot with glue in the back part of the device and tie off the knot in the front part.

2) When you have the harness well adjusted, it is advisable to hold the bird and allow it to stretch the legs.

Sometimes there is a small adjustment of the harness and you must correct it. 


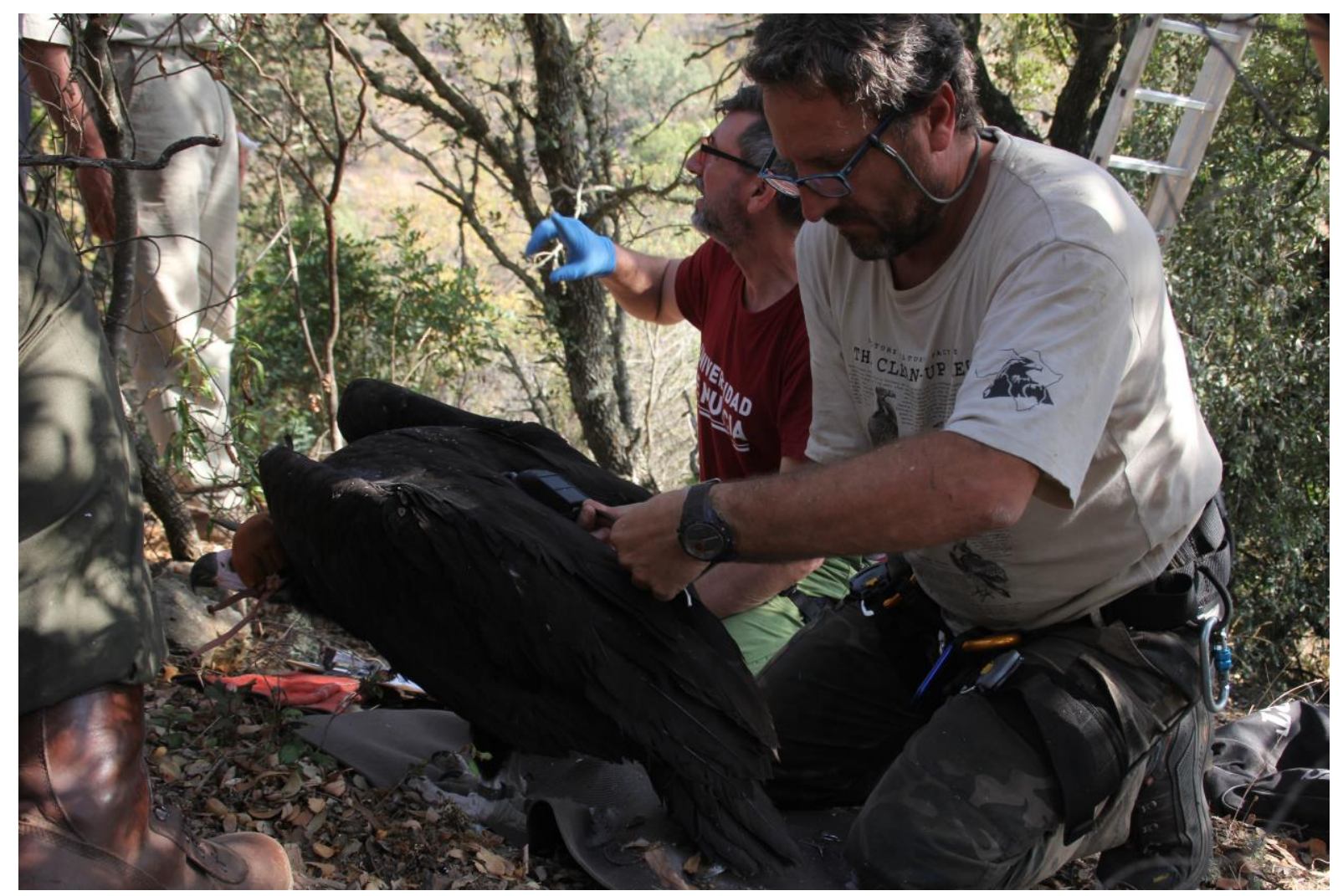

Figure 6.15

3) Check again the harness passing the fingers around the Teflon as described previously. Before you tie the knot, insert a rough/porous pad under the transmitter to prevent glue getting onto the feathers. This pad must be bigger than the device to absorb the glue that may drop by mistake. Add glue to the inner part of the ribbon before tying and remove the clamp scissors. After this, tie the knot and add again glue over it.

One knot is enough but two gives extra security. Cut the rest of the ribbon off and glue the tips to avoid fraying of the Teflon. Spray the knot and ribbon tips with cyanoacrylate activator to reduce the waiting time.
4) If you want to create a drop-off or breaking point, you can sew with floss or surgical thread both ribbons when you have them fixed with the clamp scissor. Sew 5-6 times the ribbons (or more if you want longer life of the harness on the bird) and add glue on the knot to seal it. Cut the extra ribbon and glue both tips to avoid fraying. Spray the knot and ribbon tips with cyanoacrylate activator to reduce the waiting time.

5) Remove the hood and the pad under the device and release. 


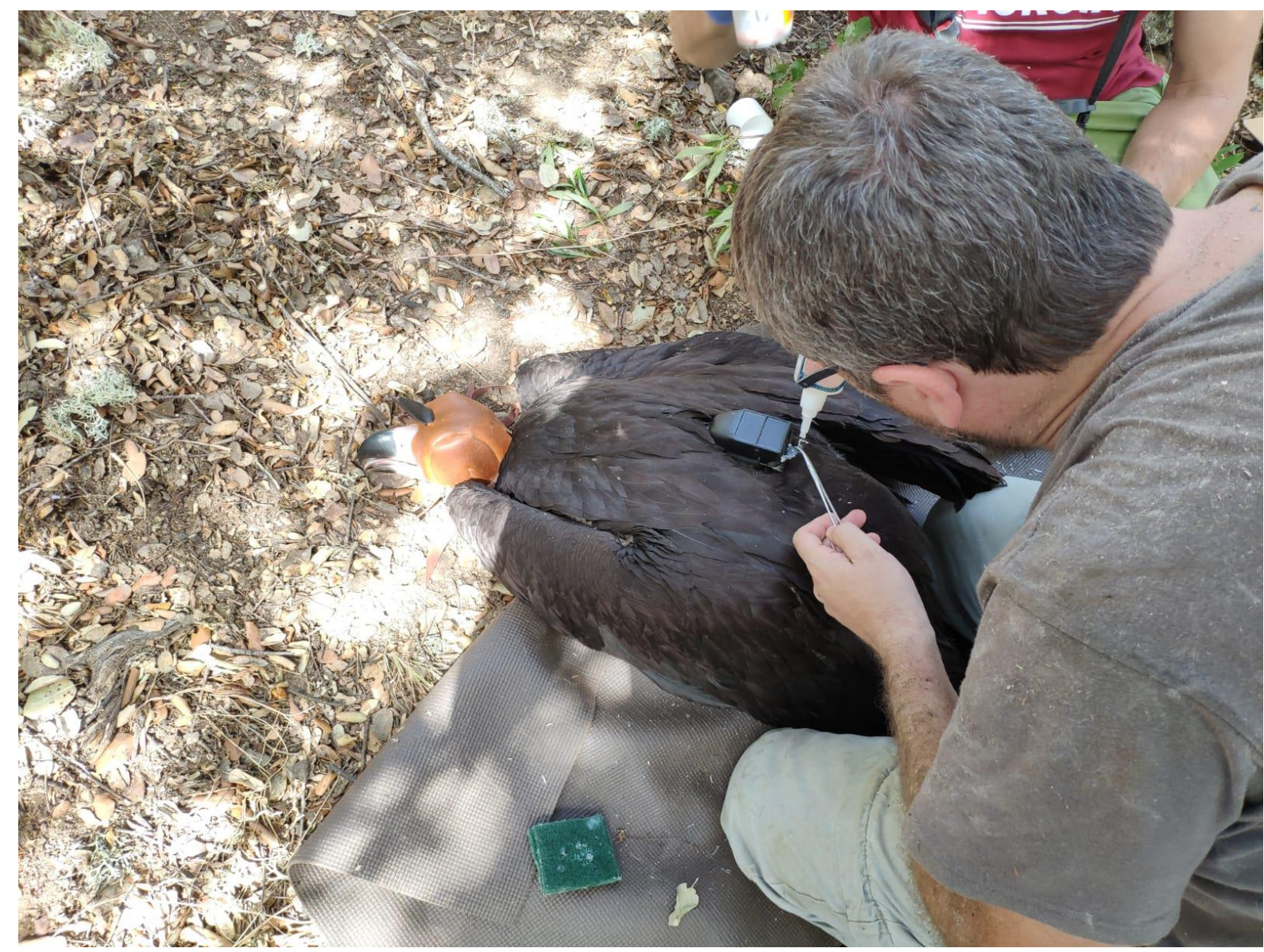

Figure 6.16

\section{Elastic harness}

1) With the elastic harness it should already be about the right size. Place the device on the back of the bird, which will be with the breast on the ground. Then, stretch one of the loops and pass the leg into the loop and repeat this operation with the other loop and leg.

2) Now the process is similar to the non-elastic harness, checking the right situation of the ribbon under the legs (no feathers in between the ribbon and the body), checking the tension of the harness and adjusting it if needed. To adjust, only tie off the knot and pull or release the elastic/ribbon until you get the proper adjustment.
3) For the final step, insert the rough pad under the device and glue inside the knot and over it after tie, cutting the extra elastic/ribbon and glue the tips.

4) Add a second knot for more security or create a drop-off or breaking point following step 4 of the non-elastic harness.

5) A stronger needle will be necessary to sew the ribbon with the elastic inside if you create a drop-off or breaking point. The use of cyanoacrylate activator will reduce the time waiting. 